\title{
MỐI QUAN HỆ GIŨ̃A CHẤT LỰ̛̣G DỊCH VỤ, SỰ HÀI LÒNG KHÁCH HÀNG VÀ LÒNG TRUNG THÀNH KHÁCH HÀNG ĐỐI VỚI CỦA HÀNG TIỆN LỘI TẠI TP. HỒ CHÍ MINH
}

\author{
ĐÀM TRÍ CƯỜNG, NGUYẼ̃N THÀNH LONG, NGUYẼ̃N NGỌC LONG, BÙI VĂN QUANG \\ Khoa Quản trị kinh doanh, Truờng Đại học Công nghiệp Thành phố Hồ Chí Minh \\ damtricuong@iuh.edu.vn
}

Tóm tắt. Nghiên cứu này nhằm xem xét mối quan hệ giữa chất lượng dịch vụ, sự hài lòng khách hàng và lòng trung thành khách hàng đối với cửa hàng tiện lợi tại TP. Hồ Chí Minh. Các mối quan hệ này được kiểm định với 1 mẫu gồm 358 khách hàng tại các cửa hàng tiện lợi trong TP. Hồ Chí Minh. Phân tích dũ liệu thông qua sử dụng mô hình cấu trúc tuyến bình phương nhỏ nhất từng phần (PLS-SEM) với phần mềm SmartPLS. Kết quả nghiên cứu cho thấy chất lượng dịch vụ có ảnh hưởng cùng chiều đến sự hài lòng khách hàng và lòng trung thành khách hàng. Kết quả nghiên cứu cũng cho thấy sự hài lòng khách hàng là biến trung gian trong mối quan hệ giữa chất lượng dịch vụ và lòng trung thành khách hàng. Từ kết quả trên, nghiên cứu đưa ra một số hàm ý cho nhà quản lý và hướng nghiên cứu tiếp theo.

Từ khóa: Chất lượng dịch vụ, sự hài lòng khách hàng, lòng trung thành khách hàng, cửa hàng tiện lợi

\section{THE RELATIONSHIP BETWEEN SERVICE QUALITY, CUSTOMER SATISFACTION AND LOYALTY FOR CONVENIENCE STORES IN HO CHI MINH CITY}

\begin{abstract}
This study aims to examine the relationship between service quality, customer satisfaction and customer loyalty for the convenience stores in Ho Chi Minh City. These relationships are tested with a sample of 358 customers at convenience stores in Ho Chi Minh City. By using the partial least squared structure equation modeling (PLS-SEM) with SmartPLS software for data analysis. The result showed that there is a positive and significant relationship between service quality on both customer satisfaction and customer loyalty. The study also showed that customer satisfaction has a partial mediation role between service quality and customer loyalty. From the above results, the study offers implications for managers and directions for future research.
\end{abstract}

Keywords. service quality, customer satisfaction, customer loyalty, convenience store

\section{GIỚI THIẸU}

Thị trường bán lẻ Việt Nam trong những năm gần đây có sự tăng trưởng nhanh chóng về quy mô, nếu như năm 2010 là 88 tỷ USD thì đến năm 2017 là 130 tỷ USD, dự báo đến năm 2020 là 179 tỷ USD [1]. Đặc biệt đối với mô hình cửa hàng tiện lợi đã có sự phát triển rất nhanh chóng về số lượng trong 5 năm gần đây, tính riêng tại TP.HCM nếu như cuối năm 2014 là 324 cửa hàng thì đến tháng $3 / 2018$ đã tăng lên 1144 cửa hàng, tăng gấp 3,5 lần; còn tính chung cả nước có khoảng 1600 cửa hàng tiện lợi [2]. Con số này sẽ còn tiếp tục tăng lên trong vài năm tiếp theo. Sự bùng nổ về số lượng đối với mô hình cửa hàng tiện lợi dẫn đến sự cạnh tranh khốc liệt đối với mô hình cửa hàng này. Bên cạnh đó, khi các nhà đầu tư trong và ngoài nước đua nhau mở chuỗi, đầu tư công nghệ, tăng cường dịch vụ để tìm chỗ đứng vững chắc tại thị trường bán lẻ Việt Nam thì sự cạnh tranh đối với mô hình cửa hàng này càng tăng. Hiện nay, mô hình cửa hàng tiện lợi này đang chứng kiến sự cạnh tranh quyết liệt của các ông lớn đã có mặt lâu năm tại thị trường như Vinmart+, Circle K, Shop \& Go, Ministop, B's Mart, Family Mart và các đại gia ngoại mới đặt chân vào Việt Nam trong 18 tháng qua như 7-Eleven, GS 25 [3]. Do đó, ngày nay trong môi trường bán lẻ cạnh tranh và năng động thì việc cung cấp dịch vụ chất lượng cao được coi là chiến lược bán lẻ cơ bản để tạo ra lợi thế cạnh tranh $[4,5]$. Ngoài ra, để duy trì khách hàng thì doanh nghiệp nên cung cấp dịch vụ tốt tại thời điểm bán hàng hoặc sau bán hàng. Chất lượng dịch vụ tốt sẽ làm hài lòng khách hàng và dẫn đến lòng trung thành khách hàng. Chất lượng được xem là cái gì đó được cảm nhận bởi khách hàng nhằm thỏa mãn kỳ vọng của họ. Dịch vụ tốt là dịch vụ có khả năng cung cấp sự hài lòng cho khách hàng hoặc có thể cung cấp dịch vụ vượt quá mong đợi của họ [6]. Một số nhà nghiên cứu cho rằng chất lượng dịch vụ ảnh hưởng đến sự hài lòng khách hàng [7-9]. Ngoài ra, chất lượng dịch vụ ảnh hưởng tích cực lên sự hài lòng khách hàng [5,10]. 
Sự hài lòng khách hàng ảnh hưởng tích cực đến lòng trung thành khách hàng [6,11]. Lòng trung thành của khách hàng được coi là chìa khóa quan trọng cho sự thành công của tổ chức và tạo ra lợi nhuận $[4,7]$.

Một vài nghiên cứu trước đây về mối quan hệ giữa chất lượng dịch vụ, sự hài lòng và lòng trung thành khách hàng trong thị trường bán lẻ nói chung $[8,10,12,13]$. Tuy nhiên, rât ít nghiên cứu phân tích mối quan hệ giữa chất lượng dịch vụ, sự hài lòng và lòng trung thành khách hàng đối với mô hình cửa hàng tiện lợi. Vì vậy, mục tiêu nghiên cứu nhằm xem xét mối quan hệ giữa chất lượng dịch vụ, sự hài lòng khách hàng và lòng trung thành khách hàng trong mô hình cửa hàng tiện lợi tại TP. Hồ Chí Minh.

Sau phần giới thiệu, các phần tiếp theo của bài viết này bao gồm phần 2 - cơ sở lý thuyết; phần 3 - phương pháp nghiên cứu; phần 4 -kết quả nghiên cứu ; phần 5 - thảo luận kết quả nghiên cứu và hàm ý quản trị; và cuối cùng, phần 6 - Hạn chế nghiên cứu và hướng nghiên cứu tiếp theo.

\section{CƠ SỞ LÝ THUYẾT VÀ MÔ HÌNH NGHIÊN CÚU}

\subsection{Chất lượng dịch vụ}

Kotler và Keller (2012) [14] cho rằng dịch vụ là bất kỳ hành động hay sự thực hiện mà một bên có thể cung cấp cho bên kia, và chủ yếu là vô hình và không dẫn đến sở hữu bất cứ thứ gì. Việc tạo ra nó có thể có, hoặc cũng có thể không gắn với một sản phẩm hữu hình. Chất lượng dịch vụ là mức độ khác biệt giữa sự mong đợi của khách hàng và cảm nhận của họ về kết quả dịch vụ [15]. Chất lượng dịch vụ là sự cảm nhận của khách hàng về kết quả dịch vụ [16]. Chất lượng dịch vụ cũng có thể được hiểu là việc chăm sóc của công ty đối với khách hàng [17]. Thang đo chất lượng dịch vụ gọi tắt là thang đo SERVQUAL, bao gồm 5 thành phần cơ bản đó là: (1) Phương tiện hữu hình (tangibles): Cơ sở vật chất, trang thiết bị, ngoại hình, trang phục của nhân viên phục vụ, (2) Tin cậy (reliability): Khả năng thực hiện dịch vụ phù hợp và đúng thời hạn ngay từ lần đầu tiên; (3) Đáp ứng (responsiveness): Sự mong muốn, sẵn sàng của nhân viên phục vụ cung cấp dịch vụ kịp thời cho khách hàng; (4) Năng lực phục vụ (assurance): Trình độ chuyên môn và cung cách phục vụ lịch sự, niềm nở với khách hàng; (5) Đồng cảm (emphathy): Sự quan tâm chăm sóc đến từng cá nhân khách hàng [15,18]. Parasuraman và cộng sự (1988) [15] đã kiểm định thang đo SERVQUAL cho nhiều ngành dịch vụ khác nhau (chẳng hạn: ngân hàng, dịch vụ thẻ tín dụng, dịch vụ điện thoại đường dài; dịch vụ sửa chữa và bảo trì). Thang đo SERVQUAL đều đạt độ tin cậy và giá trị đối với các ngành trên và có thể áp dụng thang đo này đối với các ngành dịch vụ khác [15]. Tuy nhiên, nhiều nhà nghiên cứu khác cũng đã kiểm định thang đo thang đo này với những dịch vụ khác nhau tại nhiều quốc gia. Kểt quả cho thấy các thành phần của chất lượng dịch vụ không đồng nhất ở từng ngành dịch vụ và từng thị trường khác nhau $[5,12,16,19]$

\subsection{Chất lượng dịch vụ bán lẻ}

Dabholka và cộng sự (1996) [19] kết hợp nghiên cứu định tính, lý thuyết dịch vụ và thang đo SERVQUAL đề xuất thang đo chất lượng dịch vụ bán lẻ (RSQS) gồm 5 thành phần cơ bản:

(1) Phương tiện hữu hình

(2) Tin cậy

(3) Tương tác cá nhân

(4) Giải quyết vấn đề

(5) Chính sách của cửa hàng

Dabholka và cộng sự (1996) [19] cho rằng thang đo chất lượng dịch vụ bán lẻ có thể áp dụng cửa hàng kinh doanh bán lẻ - loại hình bao gồm sản phẩm và dịch vụ.

\subsection{Các giả thuyết và mô hình nghiên cứu}

\subsubsection{Sự hài lòng khách hàng}

Sự hài lòng khách hàng là 1 vẩn đề quan trọng trong lý thuyết cũng như thực tiễn đối với hầu hết các nhà tiếp thị và nhà nghiên cứu người tiêu dùng [19]. Sự hài lòng khách hàng là cảm giác về niềm vui hoặc thất vọng của khách hàng khi so sánh kết quả cảm nhận của khách hàng về sản phẩm hoặc dịch vụ với những mong đợi của họ $[6,20,21]$. Sự hài lòng khách hàng là kết quả hoạt động marketing và dịch vụ liên quan đến quá trình mua hàng [6]. Sự hài lòng của khách hàng ngày càng trở thành mục tiêu của công ty khi ngày càng nhiều công ty phấn đấu về chất lượng trong các sản phẩm và dịch vụ của họ [22]. Do đó, sự hài lòng khách hàng có thể được coi là bản chất của sự thành công trong thế giới kinh doanh cạnh tranh cao ngày nay [5,23].

Chất lượng dịch vụ và sự hài lòng tuy là 2 khái niệm khác nhau nhưng có liên quan chặt chẽ với nhau trong nghiên cứu dịch vụ $[12,15]$. Chất lượng dịch vụ liên quan đến việc cung cấp dịch vụ, còn sự hài lòng là 
đánh giá của khách hàng sau khi sử dụng dịch vụ hay mua hàng $[8,12]$. Một số nghiên cứu trước cho thấy rằng chất lượng dịch vụ có ảnh hưởng tích cực đối với sự hài lòng khách hàng $[8,10,24]$.

Dựa trên cơ sở trên, đề xuất giả thuyết $\mathrm{H} 1$ như sau:

Giả thuyết H1: Chất lượng dịch vụ có ảnh hưởng cùng chiều đến sự hài lòng khách hàng.

\subsubsection{Lòng trung thành khách hàng}

Lòng trung thành khách hàng thường liên quan đến việc khách hàng sẵn sàng liên tục mua hàng hoặc dịch vụ với tâm lý và thái độ tốt đối với hàng hóa hoặc đối với công ty cung cấp hàng hóa hoặc dịch vụ [25-29]. Khách hàng trung thành có xu hướng mua lại sản phẩm, mua hàng trong số các dòng sản phẩm, giới thiệu sản phẩm cho người khác và có thể chỉ ra chất lượng sản phẩm của các công ty tương tự khác sản xuất [6]. Khi khách hàng đánh giá tốt về chất lượng dịch vụ thì mối quan hệ giữa cửa hàng và khách hàng trở nên gắn bó hơn. Khách hàng sẽ mua hàng lại, mua hàng nhiều hơn và có thể chấp nhận giá cao mà không chuyển sang cửa hàng khác. Điều này, dẫn đến khách hàng sẽ trung thành với cửa hàng $[12,29,30]$. Các nghiên cứu trước cho thấy rằng chất lượng dịch vụ có ảnh hưởng cùng chiều đối với lòng trung thành khách hàng [31,32].

Sự hài lòng khách hàng là quá trình gây ra lòng trung thành khách hàng, nhưng mức độ hài lòng của khách hàng có khả năng hình thành lòng trung thành là mức độ hài lòng thực sự mà các nhà cung cấp dịch vụ khác không thể cung cấp, chẳng hạn như niềm vui hoặc làm ngạc nhiên cho khách hàng $[6,33]$. Mức độ trung thành thực sự bắt đầu với việc đánh giá rất hài lòng với những gì mà khách hàng nhận được từ công ty [34]. Nguyên nhân chính của việc trở thành khách hàng trung thành là họ rất hài lòng với dịch vụ nhận được từ công ty [6]. Các nghiên cứu trước cho thấy rằng sự hài lòng của khách hàng ảnh hưởng tích cực đến lòng trung thành khách hàng $[6,8]$.

Sự hài lòng khách hàng cũng được xem như là biến trung gian trong mối quan hệ giữa chất lượng dịch vụ và lòng trung khách hàng $[35,36]$. Một số nghiên cứu trước cho thấy rằng có mối quan hệ tích cực giữa chất lượng dịch vụ, sự hài lòng khách hàng và lòng trung thành khách hàng $[35,36]$.

Dựa vào cơ sở trên, chúng tôi đề xuất giả thuyết $\mathrm{H} 2, \mathrm{H} 3, \mathrm{H} 4$ như sau:

Giả thuyết $\mathrm{H} 2$ : Chất lượng dịch vụ có ảnh hưởng cùng chiều đến lòng trung thành khách hàng.

Giả thuyết H3: Sự hài lòng khách hàng có ảnh hưởng cùng chiều đến lòng trung thành khách hàng.

Giả thuyết H4: Sự hài lòng khách hàng là trung gian trong mối quan hệ giữa chất lượng dịch vụ và lòng trung thành khách hàng.

\subsubsection{Mô hình nghiên cứu}

Mối quan hệ giữa chất lượng dịch vụ, sự hài lòng khách hàng và lòng trung thành khách hàng đối với mô hình cửa hàng tiện lợi được thể hiện qua Hình 1 :

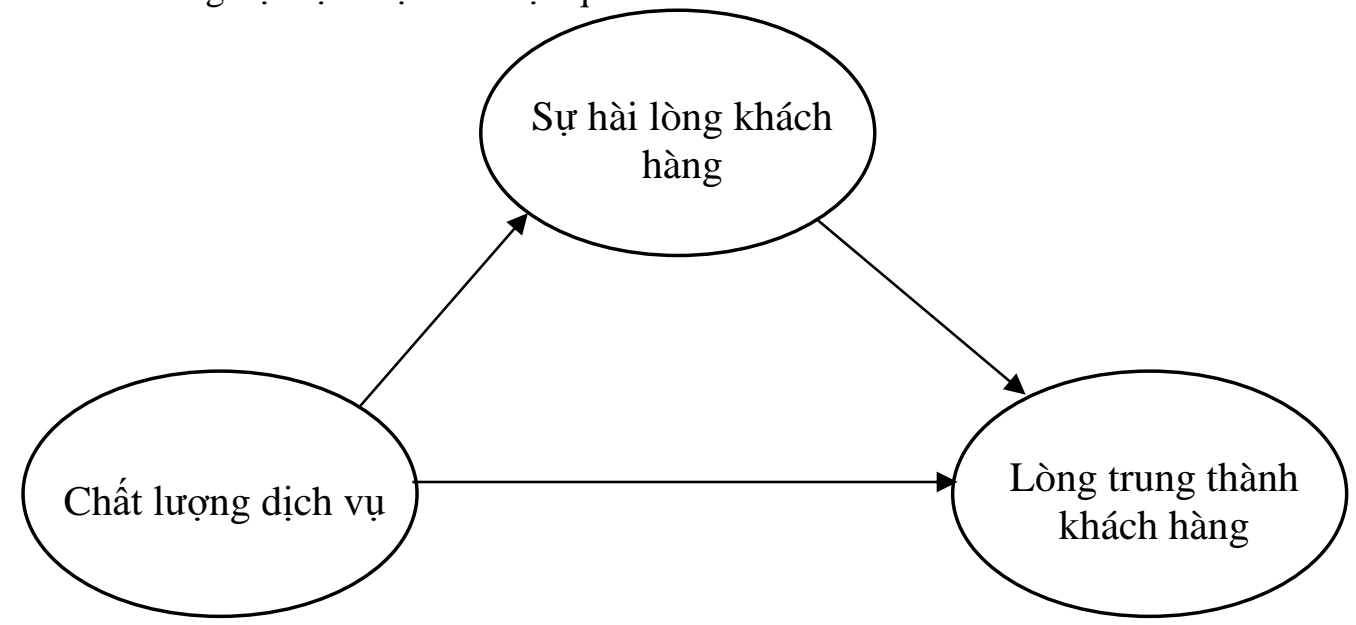

Hình 1: Mô hình nghiên cứu

\section{PHƯƠNG PHÁP NGHIÊN CÚU}

\subsection{Thang đo}

Mô hình nghiên cứu gồm 3 khái niệm: chất lượng dịch vụ, sự hài lòng khách hàng và lòng trung thành khách hàng. Thang đo lường cho các khái niệm này được kế thừa từ các nghiên cứu trước đây. Các mục hỏi được đo lường trên thang đo Likert 5 điểm với 1 là hoàn toàn không đồng ý và 5 là hoàn toàn đồng ý. 
Trong bài viết này, để đo lường vai trò trung gian của sự hài lòng khách hàng trong mối quan hệ giữa chất lượng dịch vụ và lòng trung thành khách hàng, chất lượng dịch vụ được tiếp cận như là 1 khái niệm chung. Do đó, 5 biến quan sát để đo lường khái niệm chất lượng dịch vụ chung được điều chỉnh từ thang đo SERVQUAL [15,37], thang đo SERVPERF [16] và các nghiên cứu khác về chất lượng dịch vụ [5-7]. Tuy nhiên, 5 biến quan sát này dùng để đánh giá cảm nhận của khách hàng về kết quả dịch vụ hơn là khoảng cách giữa mong đợi và cảm nhận của khách hàng về kết quả dịch vụ $[6,16,19]$. Đo lường khái niệm sự hài lòng khách hàng thể hiện qua 4 biến quan sát được điều chỉnh từ $[5,8]$. Đo lường khái niệm lòng trung thành khách hàng thể hiện qua 3 biến quan sát được điều chỉnh từ $[8,38]$.

\subsection{Thu thập dữ liệu và mẫu}

Đối tượng thu thập dữ liệu là khách hàng đã mua sắm trong các cửa hàng tiện lợi tại TP. Hồ Chí Minh. Mẫu khảo sát được tiến hành theo phương pháp thuận tiện với các nhóm khách hàng khác nhau về giới tính, tuổi tác và thu nhập trên nhiều địa điểm tại $\mathrm{TP}$. Hồ Chí Minh nhằm đạt tính đại diện cho mẫu thu thập. Trong tổng số 400 bảng câu hỏi thu thập được, có 358 bảng câu hỏi sử dụng được sau khi làm sạch dữ liệu. Đặc điểm mẫu thu thập được mô tả trong Bảng 1 .

\begin{tabular}{|c|l|c|c|}
\hline \multicolumn{4}{|c}{ Bảng 1: Đặc điểm mẫu thu thập } \\
\hline \multirow{4}{*}{ Đặc điếi tính } & Nam & Tần suất & Tỷ lệ (\%) \\
\cline { 2 - 4 } & Nữ & 83 & 23,2 \\
\cline { 2 - 4 } & Tổng & 275 & 76,8 \\
\hline \multirow{4}{*}{ Độ tuổi } & $<22$ & $\mathbf{3 5 8}$ & $\mathbf{1 0 0 , 0}$ \\
\cline { 2 - 4 } & $22-29$ & 48 & 13,4 \\
\cline { 2 - 4 } & $30-45$ & 113 & 31,6 \\
\cline { 2 - 4 } & $>45$ & 177 & 49,4 \\
\cline { 2 - 4 } & Tồng & 20 & 5,6 \\
\hline \multirow{4}{*}{$\begin{array}{c}\text { Thu nhập (triệu } \\
\text { đồng/tháng) }\end{array}$} & $<5$ & $\mathbf{3 5 8}$ & $\mathbf{1 0 0 , 0}$ \\
\cline { 2 - 4 } & $5-<10$ & 47 & 13,1 \\
\cline { 2 - 4 } & $10-<20$ & 182 & 50,8 \\
\cline { 2 - 4 } & $20-<30$ & 122 & 34,1 \\
\cline { 2 - 4 } & $>30$ & 7 & 0 \\
\cline { 2 - 4 } & Tồng & 0 & $\mathbf{1 0 0}$ \\
\hline
\end{tabular}

\subsection{Phương pháp nghiên cứu}

Nguồn: Kết quả xử lý dữ liệu thống kê của tác giả

Phương pháp nghiên cứu định lượng được thực hiện để kiểm định mô hình nghiên cứu và các giả thuyết nghiên cứu. Mẫu thu thập trước tiên được sàng lọc và loại bỏ những dữ liệu không đạt yêu cầu. Tiếp theo mô hình cấu trúc tuyến tính được sử dụng để kiểm định giả thuyết nghiên cứu. Khác với hầu hết nghiên cứu trước đây sử dụng CB-SEM (ví dụ AMOS), nghiên cứu này thực hiện mô hình cấu trúc tuyến tính bình phương tối thiểu từng phần PLS-SEM bằng phần mềm Smart PLS 3.2.7 vì phương pháp này được sử dụng rộng rãi trong các nghiên cứu hiện nay [39]. Bên cạnh đó, PLS-SEM cũng thể hiện một số ưu điểm so với CB-SEM trong 1 số trường hợp: (1) Tránh được vấn đề kích thước mẫu nhỏ và dữ liệu phân phối không chuẩn, (2) Có thể ước lượng được các mô hình phức tạp với nhiều biến tiềm ẩn và biến quan sát, (3) Có thể xử lý cả mô hình đo lường kết quả và mô hình đo lường nguyên nhân, (4) Thích hợp cho các nghiên cứu định hướng dự đoán $[39,40]$.

Kiểm định mổ hình nghiên cứu đề xuất và các giả thuyết được tiến hành thông qua hai giai đoạn: (1) Kiểm định mồ hình thang đo và (2) kiểm định mô hình cấu trúc [39]. (1) Kiểm định mô hình thang đo bao gồm (1.1) kiểm định độ tin cậy nhất quán nội tại thông qua Cronbach's Alpha và độ tin cậy tổng hợp $(\mathrm{CR})$. Hệ số độ tin cậy Cronbach's Alpha $\geq 0,7$ và độ tin cậy tổng hợp $(\mathrm{CR}) \geq 0,7$ thì thang đo đạt độ tin cậy nhất quán nội tại [39]; (1.2) kiểm định giá trị hội tụ. Hệ số tải nhân tố của các biến quan sát > 0,70 (bình phương lớn hơn 0,50 ) và phương sai trích $(\mathrm{AVE})>0,50$ thì thang đo đạt giá trị hội tụ [39,41] và $(1.3)$ kiểm định giá trị phân biệt. Giá trị phân biệt được đánh giá thông qua tiêu chí Fornell-Larcker, giá trị phân biệt đạt yêu cầu khi giá trị căn bậc hai của phương sai trích (AVE) của thang đo các khái niệm lớn hơn giá trị tương quan giữa các cặp khái niệm [42]. (2) Kiểm định mô hình cấu trúc bao gồm (2.1) kiểm định mức độ phù hợp của mô hình với dữ liệu thị trường. Để đánh giá mức độ phù hợp của mô hình với dữ liệu thị trường, 
chỉ số SRMR (Standardized Root Mean Square Residual) được xem xét. Chỉ số SRMR phải < 0,08 hoặc < 0,10 và chỉ số SRMR được xem là chỉ số goodness of fit của mô hình PLS-SEM và có thể được sử dụng để tránh hiện tượng sai lệch thông số trong mô hình [43]; (2.2) kiểm định Boostrapping dùng để đánh giá độ tin cậy của các ước lượng trong mô hình; đánh giá mối quan hệ giữa các khái niệm trong mô hình (các giả thuyết có giá trị t-value $>1,96$ thì có ý nghĩa thống kê ở mức $5 \%)$; (2.3) hệ số xác định $\left(\mathrm{R}^{2}\right)$ dùng để đo lường mức độ ảnh hưởng chung của mô hình cấu trúc như trong phân tích hồi quy [44]. Giá trị $\mathrm{R}^{2}$ gồm các giá trị 0,$19 ; 0,33$ và 0,67 tương ứng khả năng ảnh hưởng yếu, trung bình và mạnh của mô hình cấu trúc [41]. Hệ số mức độ ảnh hưởng $\left(\mathrm{f}^{2}\right)$ gồm các mức 0,$02 ; 0,15$ và 0,35 tương ứng mức độ ảnh hưởng yếu, trung bình và mạnh [45].

\section{KẾT QUẢ NGHIÊN CÚU}

\subsection{Kiểm định mô hình thang đo}

Kết quả phân tích thang đo các khái niệm nghiên cứu được trình bày trong Bảng 2.

Bảng 2: Thang đo các khái niệm nghiên cứu

\begin{tabular}{|c|c|c|c|c|c|}
\hline Khái niệm & Biến quan sát & $\begin{array}{c}\text { Hệ số tải } \\
\text { nhân tố }\end{array}$ & $\begin{array}{c}\text { Cronbach's } \\
\text { Alpha } \\
(>\mathbf{0 , 7 0}) \\
\end{array}$ & $\begin{array}{c}\text { CR } \\
(>\mathbf{0 , 7 0})\end{array}$ & $\begin{array}{c}\text { AVE } \\
(>\mathbf{0 , 5 0})\end{array}$ \\
\hline \multirow{5}{*}{$\begin{array}{l}\text { Chất lượng dịch vụ } \\
\text { (SQ) }\end{array}$} & SQ1 & 0,756 & \multirow{5}{*}{0,888} & \multirow{5}{*}{0,913} & \multirow{5}{*}{0,677} \\
\hline & SQ2 & 0,808 & & & \\
\hline & SQ3 & 0,860 & & & \\
\hline & SQ4 & 0,864 & & & \\
\hline & SQ5 & 0,802 & & & \\
\hline \multirow{4}{*}{$\begin{array}{l}\text { Sự hài lòng khách } \\
\text { hàng (SAT) }\end{array}$} & SAT1 & 0,814 & \multirow{4}{*}{0,826} & \multirow{4}{*}{0,884} & \multirow{4}{*}{0,657} \\
\hline & SAT2 & 0,811 & & & \\
\hline & SAT3 & 0,816 & & & \\
\hline & SAT4 & 0,802 & & & \\
\hline \multirow{3}{*}{$\begin{array}{l}\text { Lòng trung thành } \\
\text { khách hàng (LOY) }\end{array}$} & LOY1 & 0,848 & \multirow{3}{*}{0,843} & \multirow{3}{*}{0,905} & \multirow{3}{*}{0,762} \\
\hline & LOY2 & 0,906 & & & \\
\hline & LOY3 & 0,863 & & & \\
\hline
\end{tabular}

Nguồn: Kết quả xử lý dữ liệu của tác giả

(1) Kiểm định độ tin cậy nhất quán nội tại thông qua Cronbach's Alpha và độ tin cậy tồng hợp (CR) Kết quả phân tích thang đo các khái niệm nghiên cứu Bảng 2 cho thấy các thang đo đều có Cronbach's Alpha lớn hơn 0,70 và độ tin cậy tổng hợp $(\mathrm{CR})$ đều lớn hơn 0,70 . Do đó, các thang đo đều đạt độ tin cậy nhất quán nội tại.

\section{(2) Kiểm định giá trị hội tụ}

Kết quả phân tích thang đo các khái niệm nghiên cứu Bảng 2 cho thấy các hệ số tải nhân tố của các biến quan sát đều lớn hơn 0,70 (bình phương lớn hơn 0,50 ) và phương sai trích $(\mathrm{AVE})$ đều lớn hơn 0,50 . Do đó, các thang đo đều đạt giá trị hội tụ.

\section{(3) Kiểm định giá trị phân biệt}

Giá trị phân biệt được đánh giá thông qua tiêu chí Fornell-Larcker. Kết quả phân tích giá trị phân biệt Bảng 3 cho thấy giá trị căn bậc hai của phương sai trích (AVE) của các khái niệm lòng trung thành khách hàng, sự hài lòng khách hàng và chất lượng dịch vụ (giá trị nhỏ nhất: 0,810 ) lớn hơn giá trị tương quan giữa các cặp khái niệm (giá trị lớn nhất: 0,637 ). Do đó, các khái niệm đạt được giá trị phân biệt.

Bảng 3: Giá trị phân biệt

\begin{tabular}{|l|c|c|c|}
\hline \multicolumn{1}{|c|}{ Khái niệm } & $\begin{array}{c}\text { Lòng trung thành } \\
\text { khách hàng }\end{array}$ & $\begin{array}{c}\text { Sự hài lòng khách } \\
\text { hàng }\end{array}$ & $\begin{array}{c}\text { Chất lượng dịch } \\
\text { vụ }\end{array}$ \\
\hline Lòng trung thành khách hàng & $\mathbf{0 , 8 7 3}$ & & \\
\hline Sự hài lòng khách hàng & 0,637 & $\mathbf{0 , 8 1 0}$ & \\
\hline Chất lượng dịch vụ & 0,621 & 0,636 & $\mathbf{0 , 8 2 3}$ \\
\hline
\end{tabular}

Nguồn: Kết quả xử lý dữ liệu của tác giả 


\subsection{Kiểm định mô hình cấu trúc}

Kết quả mô hình cấu trúc SEM được trình bày ở Hình 2 và Bảng 4 cho thấy mô hình có giá trị thống kê Chi-bình phương là 414,470 với p-value $=0,000<0,005$. Nếu mô hình nhận được giá trị SRMR nhỏ hơn 0,1 thì mô hình được xem là phù hợp với dữ liệu thị trường [43]. Do đó, với kết quả phân tích có $\mathrm{SRMR}=$ $0,073<0,100$ thì mô hình nghiên cứu đề xuất phù hợp với dữ liệu thị trường. Ngoài ra, kiểm định hiện tượng đa cộng tuyến đều cho giá trị VIF < 5, nên mô hình không vi phạm hiện tượng này [39].

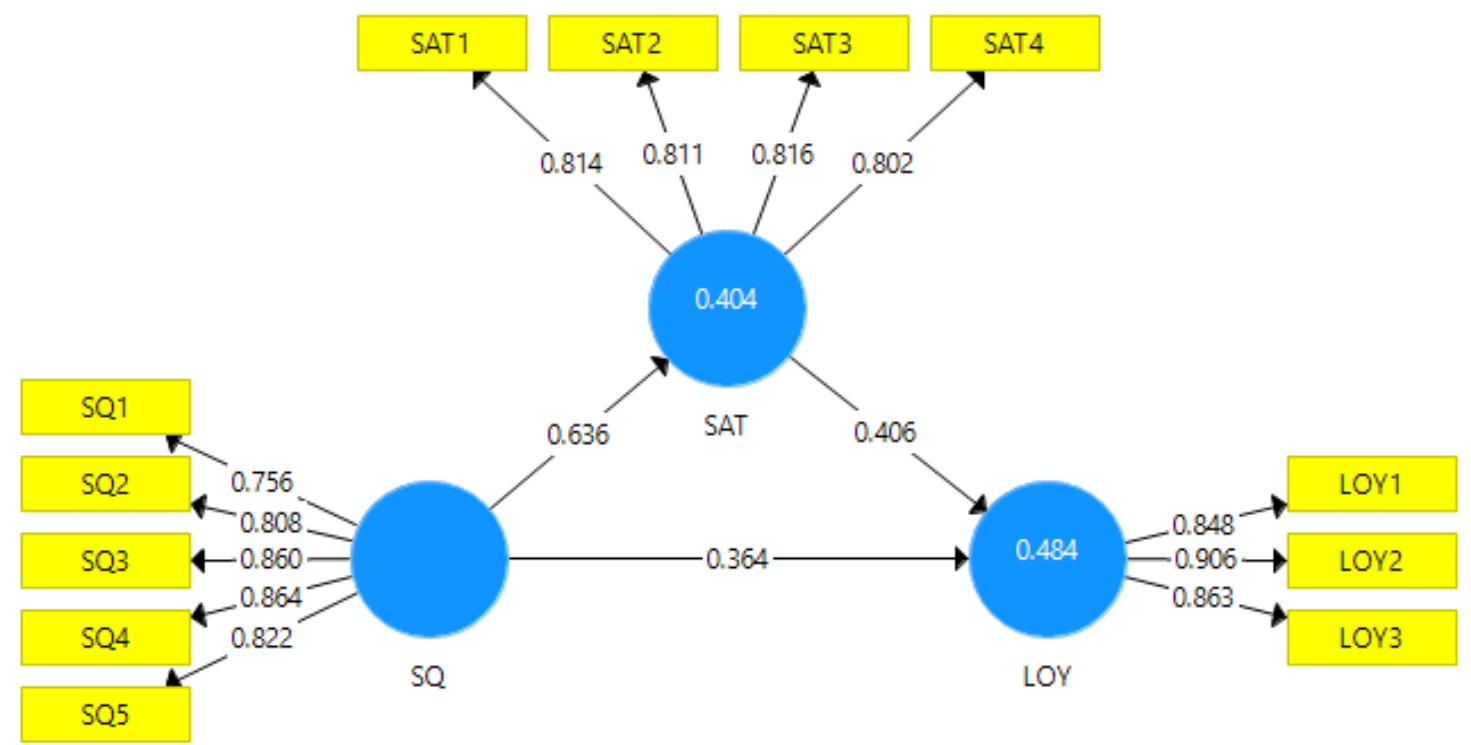

Hình 2: Mô hình cấu trúc tuyến tính PLS-SEM

Nguồn: Kết quả xử lý dữ liệu của tác giả Bảng 4: Chỉ số đo lường mức độ phù hợp mô hình với dữ liệu thị trường

\begin{tabular}{|l|c|c|}
\hline & Mô hình bão hòa & Mồ hình ước lượng \\
\hline SRMR & 0,073 & 0,073 \\
\hline d_ULS & 0,410 & 0,410 \\
\hline d_G1 & 0,187 & 0,187 \\
\hline d_G2 & 0,210 & 0,210 \\
\hline Chi-square & 414,470 & 414,470 \\
\hline NFI & 0,835 & 0,835 \\
\hline
\end{tabular}

\subsection{Kiểm định Bootstrapping}

Nguồn: Kết quả xử lý dữ liệu của tác giả

Kết quả ước lượng Bootstrapping với số lượng mẫu lặp lại 1000 quan sát cho thấy trọng số gốc có ý nghĩa với trọng số trung bình vì tất cả trọng số đều nằm trong khoảng tin cậy $95 \%$ (xem Bảng 5). Vì vậy, có thể kết luận là các ước lượng trong mồ hình đáng tin cậy.

Bảng 5: Kết quả Bootstrapping mô hình cấu trúc

\begin{tabular}{|l|c|c|c|c|c|}
\hline \multicolumn{1}{|c|}{ Mối quan hệ } & Trọng số gốc & $\begin{array}{c}\text { Trọng số } \\
\text { trung bình }\end{array}$ & Sai số chuẩn & $\mathbf{2 , 5 \%}$ & $\mathbf{9 7 , 5 \%}$ \\
\hline SQ $\rightarrow$ SAT & 0,636 & 0,637 & 0,034 & 0,571 & 0,701 \\
\hline SQ $\rightarrow$ LOY & 0,364 & 0,366 & 0,047 & 0,267 & 0,456 \\
\hline SAT $\rightarrow$ LOY & 0,406 & 0,404 & 0,047 & 0,311 & 0,496 \\
\hline
\end{tabular}

\subsection{Kiểm định giả thuyết}

Nguồn: Kết quả xử lý dữ liệu của tác giả

Kết quả ước lượng Bootstrapping mối quan hệ giữa các khái niệm nghiên cứu cho thấy các giả thuyết $\mathrm{H} 1$, $\mathrm{H} 2, \mathrm{H} 3$ trong mô hình nghiên cứu đều được chấp nhận với mức ý nghĩa thống kê tại mức $5 \%$ (p-value < $5 \%$ ). Các giả thuyết được trình bày cụ thể tại Bảng 6 . 

THÀNH KHÁCH HÀNG ĐỐI VỚI CƯA HÀNG TIỆN LỢI TẠI TP. HỒ CHÍ MINH

Bảng 6: Kết quả kiểm định các giả thuyết trong mô hình cấu trúc

\begin{tabular}{|l|c|c|c|c|c|}
\hline \multicolumn{1}{|c|}{ Mối quan hệ } & $\begin{array}{c}\text { Mức độ tác } \\
\text { động }(\boldsymbol{\beta})\end{array}$ & t-value & p-value & Giả thuyết & Kết luận \\
\hline SQ $\rightarrow$ SAT & 0,636 & 18,835 & 0,000 & H1 & Chấp nhận \\
\hline SQ $\rightarrow$ LOY & 0,364 & 7,731 & 0,000 & H2 & Chấp nhận \\
\hline SAT $\rightarrow$ LOY & 0,406 & 8,404 & 0,000 & H3 & Chấp nhận \\
\hline
\end{tabular}

Nguồn: Kết quả xử lý dữ liệu của tác giả Kết quả ước lượng Bootstrapping mối quan hệ giữa chất lượng dịch vụ, sự hài lòng khách hàng và lòng trung thành khách hàng cho thấy sự hài lòng khách hàng là trung gian trong mối quan hệ giữa chất lượng dịch vụ và lòng trung thành khách hàng có ý nghĩa thống kê tại mức 5\% (Bảng 7).

Bảng 7: Kết quả Bootstrapping của kiểm định ảnh hưởng trung gian

\begin{tabular}{|c|c|c|c|c|c|c|}
\hline Giả thuyết trung gian & $\begin{array}{c}\text { Ánh } \\
\text { hưởng } \\
\text { trụ̣c tiếp }\end{array}$ & $\begin{array}{c}\text { Ảnh } \\
\text { hưởng } \\
\text { gián tiếp }\end{array}$ & t-value & p-value & $\begin{array}{c}\text { Giả } \\
\text { thuyết }\end{array}$ & $\begin{array}{c}\text { Kết } \\
\text { luận }\end{array}$ \\
\hline $\mathrm{SQ} \rightarrow \mathrm{SAT} \rightarrow \mathrm{LOY}$ & 0,364 & 0,258 & 7,635 & 0,000 & $\mathrm{H} 4$ & $\begin{array}{c}\text { Chấp } \\
\text { nhận }\end{array}$ \\
\hline
\end{tabular}

4.5. Hệ số xác định $\left(\mathbf{R}^{2}\right)$, hệ số mức độ ảnh hưởng $\left(\mathbf{f}^{2}\right)$

Nguồn: Kết quả xử lý dữ liệu của tác giả

Giá trị hệ số xác định $\left(\mathrm{R}^{2}\right)$ được thể hiện qua Bảng 8 cho thấy mức độ tác động chung của mô hình cấu trúc là khá mạnh $\left(\mathrm{R}^{2}=48,4 \%\right)$, điều này có nghĩa là $48,4 \%$ sự biến thiên của lòng trung thành khách hàng được giải thích bởi sự hài lòng khách hàng và chất lượng dịch vụ của mô hình cấu trúc. Trong khi, chất lượng dịch vụ giải thích 40,4\% sự biến thiên của sự hài lòng khách hàng.

Bảng 8: Hệ số xác định $\left(\mathrm{R}^{2}\right)$

\begin{tabular}{|c|c|}
\hline & $\mathbf{R}^{2}$ \\
\hline LOY & 0,484 \\
\hline SAT & 0,404 \\
\hline
\end{tabular}

Nguồn: Kết quả xử lý dữ liệu của tác giả Hệ số mức độ ảnh hưởng ( $\mathrm{f}^{2}$ ) được thể hiện qua Bảng 9 cho thấy chất lượng dịch vụ có ảnh hưởng mạnh đến sự hài lòng khách hàng $(0,679)$ và có ảnh hưởng trung bình đến lòng trung thành khách hàng $(0,153)$. Trong khi, sự hài lòng khách hàng có ảnh hưởng trung bình đến lòng trung thành khách hàng $(0,190)$.

Bảng 9: Hệ số mức độ ảnh hưởng $\left(\mathrm{f}^{2}\right)$

\begin{tabular}{|c|c|c|}
\hline & \multicolumn{2}{|c|}{$\mathbf{f}^{\mathbf{2}}$} \\
\hline & LOY & SAT \\
\hline SAT & 0,190 & 0,679 \\
\hline SQ & 0,153 & 0,1 \\
\hline
\end{tabular}

Nguồn: Kết quả xử lý dữ liệu của tác giả

\section{THẢO LUẬN KẾT QUẢ NGHIÊN CÚU VÀ HÀM Ý QUẢN TRI}

\subsection{Thảo luận kết quả nghiên cứu}

Kết quả kiểm định các giả thuyết nghiên cứu tại Bảng 6 , Bảng 7 cho thấy chất lượng dịch vụ có mối quan hệ cùng chiều và tác động mạnh đến sự hài lòng khách hàng, được thể hiện qua mức độ tác động $\beta=0,636$ và $t$-value $=18,835>1,96$ và ước lượng này có ý nghĩa thống kê tại mức $5 \%$. Kết quả nghiên cứu này cũng phù hợp với các nghiên cứu thực nghiệm trước đây $[5,8]$. Chất lượng dịch vụ có mối quan hệ cùng chiều với lòng trung thành khách hàng, được thể hiện qua mức độ tác động $\beta=0,364$ và $t$-value $=7,731>1,96$ và ước lượng này có ý nghĩa thổng kê tại mức $5 \%$. Kết quả nghiên cứu này cũng phù hợp với các nghiên cứu trước $[10,35,46,47]$. Sự hài lòng khách hàng có mối quan hệ cùng với lòng trung thành khách hàng, được thể hiện qua qua mức độ tác động $\beta=0,406$ và $\mathrm{t}$-value $=8,404>1,96$ và ước lượng này có ý nghĩa thống kê tại mức $5 \%$. Kết quả nghiên cứu này cũng phù hợp với các nghiên cứu trước $[10,35,46]$. Sự hài lòng khách hàng là trung gian trong mối quan hệ giữa chất lượng dịch vụ và lòng trung thành khách hàng có ý nghĩa thống kê tại mức $5 \%$. Kết quả nghiên cứu này cũng phù hợp với những nghiên cứu trước $[35,36]$. Vì đây là nghiên cứu thực nghiệm xem xét mối quan hệ giữa chất lượng dịch vụ, sự hài lòng khách hàng và lòng trung thành khách hàng như đề xuất trong mô hình nghiên cứu. Do đó, đóng góp của nghiên cứu 
này là xem xét mối quan hệ giữa chất lượng dịch vụ, sự hài lòng khách hàng và lòng trung thành khách hàng, và kiểm định mối quan hệ của chúng trong ngữ cảnh khác so với các nghiên cứu trước đây (các nghiên cứu trước trên thế giới chẳng hạn như $[5,8,35,47] \ldots$.., nghiên cứu này xem xét và kiểm định mối quan hệ trên tại thị trường TP. Hồ Chí Minh, Việt Nam). Ngoài ra, một đóng góp khác của nghiên cứu là nghiên cứu này xem xét mối quan hệ giữa chất lượng dịch vụ, sự hài lòng khách hàng và lòng trung thành khách hàng đối với mô hình cửa hàng tiện lợi; các nghiên cứu trước đây xem xét mối quan hệ giữa chất lượng dịch vụ, sự hài lòng khách hàng và lòng trung thành khách hàng trong thị trường bán lẻ nói chung $[8,10,12,13]$.

\subsection{Hàm ý quản trị}

Kết quả nghiên cứu đưa ra một số hàm ý quản trị cho nhà quản lý cửa hàng tiện lợi như sau:

Thứ nhất, chất lượng dịch vụ là tiền đề quan trọng đối sự hài lòng khách hàng khách hàng. Chất lượng dịch vụ tốt dẫn đến làm tăng mức độ hài lòng của khách hàng đối với cửa hàng tiện lợi. Chất lượng dịch vụ cũng là tiền đề quan trọng của lòng trung thành khách hàng. Chất lượng dịch vụ tốt cũng làm tăng mức độ lòng trung thành khách hàng đối với cửa hàng tiện lợi. Như vậy, khách hàng sẽ trung thành với cửa hàng khi khách hàng hài lòng với sản phẩm/dịch vụ mà cửa hàng cung cấp. Do đó, các nhà quản lý cửa hàng tiện lợi cần lưu ý các kết quả trên trong quá trình xây dựng và thực hiện chiến lược kinh doanh của mình. Hơn nữa, để gia tăng chất lượng dịch vụ cung cấp cho khách hàng, nhà quản lý cửa hàng tiện lợi cần chú ý đến các thành phần của chất lượng dịch vụ như phương tiện hữu hình, độ tin cậy người bán, sự tương tác giữa nhân viên và khách hàng, giải quyết khiếu nại khách hàng và chính sách của cửa hàng trong vấn đề xây dựng và thực hiện chiến lược của mình.

Thứ hai, việc nhà quản lý cửa hàng hiểu được mối quan hệ giữa chất lượng dịch vụ, sự hài lòng khách hàng và lòng trung thành khách hàng sẽ giúp nhà quản lý hiểu rõ hơn cách khách hàng đánh giá chất lượng dịch vụ và cách các chiến lược dịch vụ của họ ảnh hưởng đến sự hài lòng khách hàng và lòng trung thành khách hàng là mức độ khác nhau. Trên cơ sở đó, giúp nhà quản lý cửa hàng có thể phân bổ nguồn lực một cách hiệu quả và phát triển kế hoạch hợp lý để cải thiện chất lượng dịch vụ trong tình huống kinh doanh cụ thể [7].

\section{Hạn chế và hướng nghiên cứu tiếp theo}

Nghiên cứu này có một số hạn chế. Thứ nhất, nghiên cứu này chỉ thực hiện cho 1 số cửa hàng tiện lợi tại TP. Hồ Chí Minh, chưa mang tính đại diện cho các thành phố khác tại Việt Nam. Thứ hai, nghiên cứu này chỉ xem xét mối quan hệ giữa chất lượng dịch vụ, sự hài lòng khách hàng và lòng trung thành khách hàng. Do đó, các nghiên cứu tiếp theo nên xem xét thêm các khái niệm khác trong mối quan hệ trên như giá cả, niềm tin ...

\section{TÀI LIỆU THAM KHẢO}

[1] Chu Đan. Thị trường bán lẻ Việt Nam: Quy mô lớn, cạnh tranh mạnh. 2018. Available at: http://kinhtevn.com.vn/thi-truong-ban-le-viet-nam-quy-mo-lon-canh-tranh-manh-31815.html

[2] Diễm Trang. Cửa hàng tiện lợi: Trong chán, ngoài thèm. 2018. Available at: https://vietnambiz.vn/cua-hangtien-loi-trong-chan-ngoai-them-56865.html

[3] Việt Đức. Người Việt mua sắm ở cửa hàng tiện lợi nhiều hơn siêu thị. 2018. Available at: https://news.zing.vn/nguoi-viet-mua-sam-o-cua-hang-tien-loi-nhieu-hon-sieu-thi-post891556.html

[4] Reichheld FF., Sasser WE. Zero Defections: Quality Comes to Services. Harvard Business Review. 1990; : $105-113$.

[5] Yu W., Ramanathan R. Retail service quality, corporate image and behavioural intentions: the mediating effects of customer satisfaction. International Review of Retail, Distribution and Consumer Research. 2012; 22(5): 485-505.

[6] Dimyati M., Subagio NA. Impact of Service Quality, Price, and Brand on Loyalty with the mediation of Customer Satisfaction on Pos Ekspres in East Java. Mediterranean Journal of Social Sciences. 2016; 14(3): 225-238.

[7] Shpetim C. Exploring the relationships among service quality, satisfaction, trust and store loyalty among retail customers. Journal of Competitiveness. 2012; 4(4): 16-35.

[8] Beneke J., Hayworth C., Hobson R., Mia Z. Examining the effect of retail service quality dimensions on customer satisfaction and loyalty: The case of the supermarket shopper. Acta Commercii. 2012; 12(1): 27-43. 
[9] Ishaq MI., Bhutta MH., Hamayun AA., Danish RQ., Hussain NM. Role of Corporate Image, Product Quality and Customer Value in Customer Loyalty: Intervening Effect of Customer Satisfaction. Journal of Basic and Applied Scientific Research. 2014; 4(4): 89-97.

[10] Iqbal MS., Hassan MU., Habibah U. Impact of self-service technology (SST) service quality on customer loyalty and behavioral intention: The mediating role of customer satisfaction. Cogent Business and Management. Cogent; 2018; 5(1): 1-23.

[11] Yulisetiarini D. The Effect of Relationship Marketing towards Costumer Satisfaction and Customer Loyalty on Franchised Retails in East Java. Mediterranean Journal of Social Sciences. 2015; 7(1): 333-339.

[12] Nguyễn Thị Mai Trang. Chất lượng dịch vụ, sự thỏa mãn và lòng trung thành của khách hàng siêu thị Tp.HCM. Tạp chí Phát triển KH \& CN. 2006; 9(10): 57-70.

[13] Hoa. ĐTK., Trang. NTQ., Hướng. NV., Cường TH. Phân tích các nhân tố ảnh hưởng đến lòng trung thành của khách hàng đối với dịch vụ bán lẻ của hệ thống siêu thị HAPRO Mart Hà Nội. Tạp chí Khoa học Nông Nghiệp Việt Nam. 2016; 14(8): 1295-1303.

[14] Kotler P., Keller KL. Marketing Management. 14th edn. Upper Saddle River, New Jersey: Pearson Education, Inc; 2012.

[15] Parasuraman A., Zeithaml VA., Berry LL. Servqual: A multiple-item scale for measuring consumer perceptions of service quality. Journal of Retailing. 1988; 64(1): 12-40.

[16] Cronin JJ., Taylor SA. Measuring quality: A reexamination and extention. Journal of Marketing. 1992; 56(3): $55-68$.

[17] Dimyati M. Theoretical Testing on Service Quality and Product Innovation of Small-Micro Credit Banks (a Case Study). Journal of Economics, Business and Accountancy Ventura. 2011; 14(3): 225-238.

[18] Thọ. NĐ., Trang. NTM. Nghiên cứu khoa học Marketing: Ứng dụng mô hình cấu trúc tuyến tính SEM. TP. Hồ Chí Minh: NXB Đại Học Quốc Gia TP. Hồ Chí Minh; 2008.

[19] Dabholkar PA., Thorpe DI., Rentz JO. A measure of service quality for retail stores: Scale development and validation. Journal of the Academy of Marketing Science. 1996; 24(1): 3-16.

[20] Kotler P., Armstrong G. Principles of Marketing. 16th edn. Harlow: Pearson Education Limited; 2016.

[21] Cuong DT., Long NT. Service quality, price, customer satisfaction and customer loyalty: A study of convenience stores in Ho Chi Minh city. Journal of Science and Technology. 2019; 37: 25-35.

[22] Das A., Kumar V., Saha GC. Retail service quality in context of CIS countries. International Journal of Quality and Reliability Management. 2010; 27(6): 658-683.

[23] Jamal A., Naser K. Customer satisfaction and retail banking: An assessment of some of the key antecedents of customer satisfaction in retail banking. International Journal of Bank Marketing. 2002; 20(4): 146-160.

[24] Kaura V., Prasad CSD., Sharma S. Service quality, service convenience, price and fairness, customer loyalty, and the mediating role of customer satisfaction. International Journal of Bank Marketing. 2015; 33(4): 404422 .

[25] Cuong DT., Khoi BH. The Relationship between Service Quality, Satisfaction , Trust and Customer Loyalty A Study of Convenience Stores in Vietnam. Journal of Advanced Research in Dynamic and Control Systems. 2019; 11: 327-333.

[26] Deng Z., Lu Y., Wei KK., Zhang J. Understanding customer satisfaction and loyalty: An empirical study of mobile instant messages in China. International Journal of Information Management. Elsevier Ltd; 2010; 30(4): 289-300.

[27] Bakti IGMY., Sumaedi S. An analysis of library customer loyalty:The role of service quality and customer satisfaction, a case study in Indonesia. Library Management. 2013; 34(6-7): 397-414.

[28] Yeo GT., Thai V V., Roh SY. An Analysis of Port Service Quality and Customer Satisfaction: The Case of Korean Container Ports. Asian Journal of Shipping and Logistics. Elsevier B.V.; 2015; 31(4): 437-447.

[29] Ismail A., Naser K. Service quality as a predictor of customer satisfaction and customer loyalty. LogForum. 2016; 12(4): 269-283. 

THÀNH KHÁCH HÀNG ĐỐI VỚI CỦA HÀNG TIỆN LỢI TẠI TP. HỒ CHÍ MINH

[30] Zeithaml VA., Berry LL., Parasuraman A. The behavioral consequences of service quality. Journal of Marketing. 1996; 60: 31-46.

[31] Saura IG., Francés DS., Contrí GB., Blasco MF. Logistics service quality: A new way to loyalty. Industrial Management and Data Systems. 2008; 108(5): 650-668.

[32] Kuo YF., Wu CM., Deng WJ. The relationships among service quality, perceived value, customer satisfaction, and post-purchase intention in mobile value-added services. Computers in Human Behavior. Elsevier Ltd; 2009; 25(4): 887-896.

[33] Oliver RL. Satisfaction: A Behavioral Perspective on the Consumer. New York: The McGraw-Hill Companies, Inc; 1997.

[34] Jones TO., Sasser WE. Why satisfied customer defect. Harvard Business Review. 1995; 73(6): 88-99.

[35] Hadi NU., Abdullah N., Sentosa I. Making sense of mediating analysis: A marketing perspective. Review of Integrative Business and Economics ResearchOnlineCDROM. 2016; 5(2): 62-76.

[36] Sugiati T. The mediation role of customer satisfaction on loyalty. Journal of Engineering and Applied Sciences. 2017. pp. 4715-4719.

[37] Parasuraman A., Berry LL., Zeithaml VA. Refinement and reassessment of the SERVQUAL scale. 1991. pp. $420-450$.

[38] Joudeh JMM., Dandis AO. Service Quality, Customer Satisfaction and Loyalty in an Internet Service Providers. International Journal of Business and Management. 2018; 13(8): 108.

[39] Hair JF., Hult GTM., Ringle CM., Sarstedt M. A Primer on Partial Least Squares Structural Equation Modeling (PLS-SEM). 2nd edn. Los Angeles: SAGE Publications, Inc; 2017.

[40] Henseler J., Ringle CM., Sarstedt M. Using partial least squares path modeling in international advertising research: Basic concepts and recent issues. In: Okzaki S (ed) (ed.) Handbook of Partial Least Squares: Concepts, methods and applications in marketing and related fields. Berlin: Springe; 2012. pp. 252-276.

[41] Chin WW. The partial least squares approach to structural equation modeling. In: Macoulides GA (ed.) Modern Methods for Business Research. New Jersey: Lawrence Erlbaum Associates; 1998. pp. 295-336.

[42] Fornell C., Larcker DF. Evaluating Structural Equation Models with Unobservable Variables and Measurement Error. Journal of Marketing Research. 1981; 18(1): 39.

[43] Hu LT., Bentler P. Cutoff Criteria for Fit Indexes in Covariance Structure Analysis: Conventional Criteria Versus New Alternatives, Structural Equation Modeling. Structural Equation Modeling. 1999; 6(1): 1-55.

[44] Garson GD. Partial least squares: Regression \& structural equation models. 2016 ed. Asheboro: Statistical Associates Publishers; 2016. 1-262 p.

[45] Cohen J. Statistical power analysis for the behavioral sciences. New Jersey: Lawrence Erlbaum Associates; 1988.

[46] Yaya LHP., Marimon F., Casadesus M. Assessing the Effects of Online Service Quality on Loyalty and the Mediation Role of Customer. 8th International Quality Conference. 2014. pp. 223-234.

[47] Cules E., Agung AA., Kusuma GAT., Sudia IN. Retail quality model construction for customer satisfaction and loyalty: A study of retail business in Bali Indonesia. International Journal of Economics, Commerce and Management. 2018; VI(9): 29-49.

Ngày nhận bài: 03/01/2019

Ngày chấp nhận đăng: 24/10/2019 